Revista de Metalurgia 55(3)

Julio-Septiembre 2019, 55(3), e149

ISSN-L: 0034-8570

https://doi.org/10.3989/revmetalm.149

\title{
Lixiviación carbonato amoniacal: estimación del níquel disuelto en el efluente de destilación
}

\author{
Armando Rojas Vargas $^{\mathrm{a}, \mathbb{} \text {, }}$, María E. Magaña Haynes ${ }^{\mathrm{b}}$, Aymara R. Riverón ${ }^{\mathrm{b}}$ \\ a'Empresa de Servicios Técnicos de Computación, Comunicaciones y Electrónica
"Rafael Fausto Orejón Forment", Calle 1ra. No. 143 Rolo Monterrey, Moa, Holguin, Cuba \\ 'Centro de Investigaciones del Níquel "Alberto Fernández Montes de Oca", \\ ( $₫$ utor para la correspondencia: arojas@eros.moa.minem.cu)
}

Enviado: 19 Enero 2019; Aceptado: 28 Mayo 2019; Publicado on-Line: 20 Septiembre 2019

\begin{abstract}
RESUMEN: Se identificaron los factores que inciden en el incremento de la concentración del níquel disuelto en la suspensión de carbonato básico de níquel, de la tecnología de lixiviación de carbonato amoniacal. Se propuso un modelo estadístico para determinar el níquel en función de los factores: concentración de amoníaco, dióxido de carbono y azufre en la solución que alimenta a las columnas de la destilación, y el pH de la suspensión. El modelo se validó con antecedentes experimentales y una serie de ecuaciones del equilibrio termodinámico en el sistema $\mathrm{Ni}$ (II)- $\mathrm{NH}_{3}-\mathrm{CO}_{3}{ }^{2-}-\mathrm{S}_{2} \mathrm{O}_{3}{ }^{2-}-\mathrm{SO}_{4}{ }^{2-}-\mathrm{H}_{2} \mathrm{O}$. Se obtuvo que a mayor relación de $\mathrm{Ni} / \mathrm{S}$ y $\mathrm{CO}_{2} / \mathrm{S}$, y menor de $\mathrm{NH}_{3} / \mathrm{CO}_{2}$, disminuye el níquel disuelto. Cuando la alcalinidad de la suspensión disminuye a $\mathrm{pH}$ inferior a 9 , hay una tendencia al incremento de la concentración de níquel disuelto. El análisis permitió formular las posibles reacciones químicas involucradas en la lixiviación del carbonato que propician el incremento del níquel disuelto.
\end{abstract}

PALABRAS CLAVE: Carbonato básico de níquel; Destilación; Lixiviación carbonato amoniacal; Níquel disuelto; Proceso Caron

Citar como/Citation: Rojas Vargas, A.; Magaña Haynes, M.E.; Ricardo Riverón, A. (2019). "Lixiviación carbonato amoniacal: estimación del níquel disuelto en el efluente de destilación". Rev. Metal. 55(3): e149.https://doi.org/10.3989/ revmetalm.149

ABSTRACT: Ammoniacal Carbonate Leaching: Estimation of dissolved nickel in the distillation effluent. The factors that incise in the increment of the nickel dissolved concentration, in the basic nickel carbonate suspension, of the ammoniacal carbonate leaching technology were identified. A statistical model was proposed to estimate the nickel in function of the factors: ammonia concentration, carbon dioxide and sulfur in the liquor fed to the distillation columns, and the suspension $\mathrm{pH}$. The model was validated with experimental antecedents and a series of equations of the thermodynamic equilibrium in $\mathrm{Ni}$ (II)- $-\mathrm{NH}_{3}-\mathrm{CO}_{3}{ }^{2-}-\mathrm{S}_{2} \mathrm{O}_{3}{ }^{2-}-\mathrm{SO}_{4}{ }^{2-}-\mathrm{H}_{2} \mathrm{O}$ system. It was obtained that to more relationship Ni/S, $\mathrm{CO}_{2} / \mathrm{S}$ and smaller $\mathrm{NH}_{3} / \mathrm{CO}_{2}$, the dissolved nickel diminishes. When the alkalinity of the suspension diminishes to a $\mathrm{pH}$ inferior to 9 , there is a tendency to the increment of dissolved nickel concentration. The analysis allowed the formulation of the possible chemical reactions involved in the carbonate leaching, which propitiate the increment of dissolved nickel.

KEYWORDS: Ammoniacal carbonate leaching; Basic nickel carbonate; Caron process; Dissolved nickel; Distillation

ORCID ID: Armando Rojas Vargas (https://orcid.org/0000-0002-8927-2023); María E. Magaña Haynes (http://orcid. org/0000-0003-2023-8879); Aymara Ricardo Riverón (http://orcid.org/0000-0002-2014-4314)

Copyright: (C) 2019 CSIC. Este es un artículo de acceso abierto distribuido bajo los términos de la licencia de uso y distribución Creative Commons Reconocimiento 4.0 Internacional (CC BY 4.0). 


\section{INTRODUCCIÓN}

La tecnología de lixiviación de carbonato amoniacal (TCA) para la extracción selectiva de níquel y cobalto, también conocida como proceso Caron, fue desarrollada en 1940 para procesar las lateritas a presión atmosférica. En Cuba se realiza a través de las operaciones fundamentales: secado, molienda, reducción, lixiviación, destilación, absorción, calcinación y sinterización; y se produce el óxido de níquel. El cobalto se obtiene por precipitación química con el ácido sulfhídrico en un reactor tipo flujo pistón.

Se denomina Licor Producto a la solución carbonato - amoniacal, enriquecida en níquel disuelto procedente del proceso de lixiviación, posterior a la separación del cobalto.

En las columnas de destilación, al poner en contacto directo el Licor Producto con el vapor de agua a contracorriente, precipita el níquel en forma de carbonato básico de níquel (CBN), aunque también se han identificado sulfato, hidróxido y óxido del metal, Ecs. (1) y (2):

$$
\begin{aligned}
& 5 \mathrm{Ni}\left(\mathrm{NH}_{3}\right)_{6}^{2+}+5 \mathrm{CO}_{3}^{2-}+7 \mathrm{H}_{2} \mathrm{O} \rightarrow 2 \mathrm{NiCO}_{3} \\
& 3 \mathrm{Ni}(\mathrm{OH})_{2} \cdot 4 \mathrm{H}_{2} \mathrm{O}_{(s)}+3 \mathrm{CO}_{2(g)}+30 \mathrm{NH}_{3(g)}
\end{aligned}
$$

$$
\begin{aligned}
& 6 \mathrm{Ni}\left(\mathrm{NH}_{3}\right)_{6}^{2+}+5 \mathrm{CO}_{3}^{2-}+\mathrm{SO}_{4}^{2-}+3 \mathrm{H}_{2} \mathrm{O} \rightarrow 2 \mathrm{NiCO}_{3} . \\
& 3 \mathrm{Ni}(\mathrm{OH})_{2} \cdot \mathrm{NiSO}_{4(s)}+3 \mathrm{CO}_{2(g)}+36 \mathrm{NH}_{3(g)}
\end{aligned}
$$

Por el tope de la columna se desprende amoníaco y dióxido de carbono, los cuales se absorben con agua y devuelven al proceso; por el fondo, se obtiene una suspensión a una temperatura entre $80{ }^{\circ} \mathrm{C}$ y $90{ }^{\circ} \mathrm{C}$, concentración en peso entre 2,5 y $4,0 \%$, con varias especies en disolución. El níquel disuelto remanente precipita por reacción química con $\mathrm{H}_{2} \mathrm{~S}$ (García y Rojas-Vargas, 2006).

En esta etapa de destilación, se han introducido mejoras tecnológicas para disminuir el níquel y amoníaco disueltos en el efluente, y se ha logrado obtener un carbonato de mayor diámetro promedio de partícula, enriquecido en dióxido de carbono, con menos azufre, inferior humedad remanente después de la filtración, contribuyendo a reducir los gastos por precipitación química y el consumo energético en el proceso de calcinación (Rojas-Vargas y Trujillo, 2012; Magaña Haynes y Rojas-Vargas, 2013; Magaña Haynes, 2016).

Entre las mejoras obtenidas se destacan: el control complementario de la destilación por determinación de amoníaco en el plato controlante de la columna, en la planta productora en Nicaro, 1977; dosificación de $\mathrm{CBN}$ al $10 \%$ en peso de sólidos por un plato lateral de las columnas, en 1989; alimentación de solución amoniacal con alto $\mathrm{CO}_{2}$ por un plato lateral, en Nicaro, 2001 y en Punta Gorda, 2016.

El control complementario de la destilación por $\mathrm{pH}$, se evaluó en ambas plantas productoras de níquel, en Nicaro y Punta Gorda. Consiste en operar las columnas controlando los flujos de Licor Producto o vapor alimentados, de tal forma que la suspensión de CBN cumpla con cierta norma de $\mathrm{pH}$, obtenida a partir de optimizar las funciones de concentración de níquel y amoníaco, polinomial y potencial, respectivamente (Rojas-Vargas y Trujillo, 2012).

El propósito de este trabajo fue determinar un modelo para estimar la concentración de níquel disuelto en los efluentes de la destilación en el rango de $\mathrm{pH}$ entre 8 y 9 , y analizar la influencia de las variables independientes sobre la concentración.

\section{MATERIALES Y MÉTODOS}

El muestreo se realizó en las plantas productoras de níquel en Nicaro y Punta Gorda, en varios periodos o campañas para garantizar la representatividad en la composición iónica de la solución procesada en las columnas de destilación. Las muestras se tomaron en la forma que ocurren normalmente, en el Licor Producto, en el plato controlante y en la suspensión de CBN, cada $4 \mathrm{~h}$ en frascos de $100 \mathrm{~mL}$.

Las columnas de destilación industrial en Nicaro tienen diámetro $3,4 \mathrm{~m}$, altura $18 \mathrm{~m}, 18$ platos con copas de burbujeo en la proporción 24 copas/plato y presión de operación en el fondo de 127,5 MPa. En Punta Gorda, las columnas presentan un diámetro $3,0 \mathrm{~m}$, altura $27,15 \mathrm{~m}, 16$ platos con una copa de burbujeo cada uno y presión en el fondo de 49,0 $\mathrm{MPa}$.

Los análisis químicos se realizaron en el laboratorio de la Unidad de Proyecto Nicaro, Centro de Investigaciones del Níquel. Se aplicaron métodos volumétricos, gravimétricos, potenciométricos y espectrofotometría de Absorción Atómica. El pH se determinó utilizando un $\mathrm{pH}$ metro PHILIPS PW-9420, 115-230 V, 50-60 Hz.

Considerando que la composición iónica del Licor Producto alimentado a las columnas y el $\mathrm{pH}$ del efluente determinan la estructura y composición química del $\mathrm{CBN}$, y que el $\mathrm{pH}$ influye además sobre el producto de solubilidad, en la especiación de los compuestos disueltos en la suspensión de CBN y en la capacidad de lixiviación del precipitado, se tomaron como variables:

Variable dependiente: Concentración de níquel disuelto.

Variables independientes: Relación $\mathrm{NH}_{3} / \mathrm{CO}_{2}$ entre 1,5 a 2,0 ; relación $\mathrm{Ni} / \mathrm{S}$ entre 1,0 y 2,0 y la concentración de azufre (S) entre 3,5 y $5,0 \mathrm{~g} \cdot \mathrm{l}^{-1}$.

Variables fijas: Flujo del Licor Producto (Qa), temperatura en el tope de la columna (Tp), y cociente entre el flujo del licor y el flujo de vapor (Qa/Wv). 
En Nicaro, las condiciones de operación, expresadas como promedio \pm deviación estándar fueron: Qa $56,4 \mathrm{~m}^{3} \cdot \mathrm{h}^{-1}( \pm 4,5) ; \mathrm{Tp} 83,0^{\circ} \mathrm{C}( \pm 1,4)$ y Qa/Wv $5,7 \mathrm{~m}^{3} \cdot \mathrm{kg}^{-1}( \pm 1,2)$. Por su parte, en Punta Gorda, Qa $62,0 \mathrm{~m}^{3} \cdot \mathrm{h}^{-1}( \pm 3,3)$; Tp $79,4{ }^{\circ} \mathrm{C}( \pm 0,7)$ y $\mathrm{Qa} / \mathrm{Wv}$ $4,3 \mathrm{~m}^{3} \cdot \mathrm{kg}^{-1}( \pm 0,7)$. Estos valores se tomaron desde el puesto de supervisión y control, del SCADA EROS y el SCADA CITED de ambas plantas, respectivamente.

El modelo de pronóstico de la concentración de níquel disuelto en la suspensión de $\mathrm{CBN}$, se obtuvo aplicando el Statgraphics 5.1, según el polinomio de segundo orden representado por la Ec. (3). (Yoosefian et al., 2017).

$Y=k_{0}+\sum_{i=1}^{n} k_{i} x_{i}+\sum_{i=1}^{n} k_{i i} x_{i}^{2}+\sum_{i=1}^{n-1} \sum_{j=i+1}^{n} k_{i j} x_{i} x_{j}$

donde: $k_{0}, k_{i}, k_{i i}, k_{i j}$ son las constantes del modelo: intercepto, coeficiente lineal, coeficiente cuadrático y coeficiente de interacción; $x_{i} x_{j}$ representan las variables independientes; $Y$ es la variable dependiente.
El modelo se validó con antecedentes experimentales y con el algoritmo reportado por Guo et al. (2004) y Zhan et al. (2011), considerando los aniones tiosulfato $\left[\mathrm{S}_{2} \mathrm{O}_{3}{ }^{2-}\right]$ y sulfato $\left[\mathrm{SO}_{4}{ }^{2-}\right]$, las reacciones químicas (Ecs. (4) - (18)) y las constantes de equilibrio para el sistema $\mathrm{Ni}$ (II)- $\mathrm{NH}_{3}-\mathrm{CO}_{3}{ }^{2-}-\mathrm{S}_{2} \mathrm{O}_{3}{ }^{2-}$ $\mathrm{SO}_{4}{ }^{2-}-\mathrm{H}_{2} \mathrm{O}$ se muestran en la Tabla 1 .

Los complejos de níquel se representan por la reacción Ec. (19) y la expresión de la constante de estabilidad (Kn) Ec. (20), donde $M e$ es el metal, $Z$ grado de oxidación, $L$ el ligando, $n$ coeficiente estequiométrico o cantidad de sustancia, $m$ grado de oxidación del ion complejo (Ver Tabla 2).

$$
\begin{gathered}
M e^{z+}+n L \leftrightarrow\left[M e L_{n}\right]^{m} \\
K n=\beta n=\frac{\left[M e L_{n}\right]^{m}}{[M e]^{z+} \cdot[L]^{n}}
\end{gathered}
$$

La concentración de níquel (II) se estima planteando el producto iónico del agua para el hidróxido de níquel y el carbonato de níquel (Guo et al., 2004; Zhan et al., 2011), Ec. (21):

\begin{tabular}{|c|c|c|c|c|}
\hline $\mathbf{N}^{\circ}$ & Reacción química & Expresión de la constante de equilibrio & $\log \left(K_{x}\right) 25^{\circ} \mathrm{C}$ & Fuente \\
\hline (4) & $\mathrm{H}_{2} \mathrm{O} \leftrightarrow \mathrm{H}^{+}+\mathrm{OH}^{-}$ & $K w=\left[H^{+}\right] \cdot\left[\mathrm{OH}^{-}\right]$ & -14 & Guo et al. (2004) \\
\hline (5) & $N H_{3(g)} \leftrightarrow N H_{3_{(a c)}}$ & - & & \\
\hline (6) & $\mathrm{NH}_{3_{(g)}}+\mathrm{H}_{2} \mathrm{O} \leftrightarrow \mathrm{NH}_{4} \mathrm{OH}$ & $K_{H a}=\left[N_{4} O H\right] \cdot P_{N H 3}^{-1}$ & 1,76 & Scott y Hobbs (1967) \\
\hline (7) & $N H_{4}^{+} \leftrightarrow N H_{3_{(a c)}}+H^{+}$ & $\mathrm{Ka}=\left[\mathrm{NH}_{3}\right] \cdot\left[\mathrm{H}^{+}\right] \cdot\left[\mathrm{NH}_{4}^{+}\right]^{-1}$ & $-9,24$ & Zhan et al. (2011) \\
\hline (8) & $\mathrm{NH}_{4} \mathrm{OH} \leftrightarrow \mathrm{NH}_{4}^{+}+\mathrm{OH}^{-}$ & $\mathrm{Kb}=\left[\mathrm{OH}^{-}\right] \cdot\left[\mathrm{NH}_{4}^{+}\right] \cdot\left[\mathrm{NH}_{4} \mathrm{OH}\right]^{-1}$ & $-4,76$ & Scott y Hobbs (1967) \\
\hline (9) & $\mathrm{CO}_{2_{(g)}} \leftrightarrow \mathrm{CO}_{2_{(a c)}}$ & . & & \\
\hline (10) & $\mathrm{CO}_{2(a c)}+\mathrm{H}_{2} \mathrm{O} \leftrightarrow \mathrm{H}_{2} \mathrm{CO}_{3}$ & $K_{H C}=\left[\mathrm{H}_{2} \mathrm{CO}_{3}\right] \cdot P_{\mathrm{CO} 2}^{-1}$ & $-1,47$ & Scott y Hobbs (1967) \\
\hline (11) & $\mathrm{H}_{2} \mathrm{CO}_{3} \leftrightarrow \mathrm{HCO}_{3}^{-}+\mathrm{H}^{+}$ & $K_{1 c}=\left[H^{+}\right] \cdot\left[\mathrm{HCO}_{3}^{-}\right] \cdot\left[\mathrm{H}_{2} \mathrm{CO}_{3}\right]^{-1}$ & $-6,36$ & Edwards et al. (1978) \\
\hline (12) & $\mathrm{HCO}_{3}^{-} \leftrightarrow \mathrm{CO}_{3}^{2-}+\mathrm{H}^{+}$ & $K_{2 c}=\left[\mathrm{H}^{+}\right] \cdot\left[\mathrm{CO}_{3}^{2-}\right] \cdot\left[\mathrm{HCO}_{3}^{-}\right]^{-1}$ & $-10,33$ & Edwards et al. (1978) \\
\hline (13) & $\mathrm{NH}_{3}+\mathrm{HCO}_{3}^{-} \leftrightarrow \mathrm{H}_{2} \mathrm{NOO}^{-}+\mathrm{H}_{2} \mathrm{O}$ & $K_{N C}=\left[\mathrm{H}_{2} \mathrm{NOO}^{-}\right] \cdot\left[\mathrm{NH}_{3}\right]^{-1} \cdot\left[\mathrm{HCO}_{3}^{-}\right]^{-1}$ & 0,48 & Guo et al. (2004) \\
\hline (14) & $\mathrm{NiCO}_{3(s)} \leftrightarrow\left[\mathrm{Ni}^{2+}\right]+\left[\mathrm{CO}_{3}^{2-}\right]$ & $\mathrm{Kps}=\left[\mathrm{Ni}^{2+}\right] \cdot\left[\mathrm{CO}_{3}^{2-}\right]$ & $-8,18$ & Guo et al. (2004) \\
\hline (15) & $\mathrm{Ni}(\mathrm{OH})^{+} \leftrightarrow\left[\mathrm{Ni}^{2+}\right]+\left[\mathrm{OH}^{-}\right]$ & $K_{1 O}=\left[\mathrm{Ni}^{2+}\right] \cdot\left[\mathrm{OH}^{-}\right] \cdot\left[\mathrm{Ni}(\mathrm{OH})^{+}\right]^{-1}$ & $-4,97$ & Zhan et al. (2011) \\
\hline (16) & $\mathrm{Ni}(\mathrm{OH})_{2} \leftrightarrow\left[\mathrm{Ni}^{2+}\right]+\left[2 \mathrm{OH}^{-}\right]$ & $K_{2 O}=\left[\mathrm{Ni}^{2+}\right] \cdot\left[\mathrm{OH}^{-}\right]^{2} \cdot\left[\mathrm{Ni}(\mathrm{OH})_{2}\right]^{-1}$ & $-8,55$ & Zhan et al. (2011) \\
\hline (17) & $\mathrm{Ni}(\mathrm{OH})_{2(s)} \leftrightarrow\left[\mathrm{Ni}^{2+}\right]+\left[2 \mathrm{OH}^{-}\right]$ & $\mathrm{Kps}=\left[\mathrm{Ni}^{2+}\right] \cdot\left[\mathrm{OH}^{-}\right]^{2}$ & $-14,7$ & Zhan et al. (2011) \\
\hline (18) & $\mathrm{Ni}(\mathrm{OH})_{3}^{-} \leftrightarrow\left[\mathrm{Ni}^{2+}\right]+\left[3 \mathrm{OH}^{-}\right]$ & $K_{30}=\left[\mathrm{Ni}^{2+}\right] \cdot\left[\mathrm{OH}^{-}\right]^{3} \cdot\left[\mathrm{Ni}(\mathrm{OH})_{3}^{-}\right]^{-1}$ & $-11,3$ & Zhan et al. (2011) \\
\hline
\end{tabular}

TABLA 1. Reacciones y constante de equilibrio químico. Sistema $\mathrm{NH}_{3}-\mathrm{SO}_{2}-\mathrm{CO}_{2}-\mathrm{H}_{2} \mathrm{O}$. 
TABla 2. Constante de estabilidad a $25^{\circ} \mathrm{C}$ de los compuestos complejos.

\begin{tabular}{|c|c|c|c|c|c|c|c|}
\hline \multirow[b]{3}{*}{ Complejos } & \multicolumn{7}{|c|}{$\mathbf{n}$} \\
\hline & 1 & 2 & 3 & 4 & 5 & 6 & Fuente \\
\hline & \multicolumn{7}{|c|}{ Log Kn } \\
\hline \multirow{3}{*}[\mathrm{Ni}(\mathrm{NH}_{3})_{n}]{$^{2+}$} & 2,81 & 5,08 & 6,85 & 8,12 & 8,93 & 9,08 & Smith y Martel (1976) \\
\hline & 2,70 & 4,90 & 6,50 & 7,60 & 8,30 & 8,74 & Hummel et al. (2014) \\
\hline & 2,80 & 5,04 & 6,77 & 7,96 & 8,71 & 8,74 & Zhan et al. (2011) \\
\hline \multirow{2}{*}[\mathrm{Ni}(\mathrm{S}_{2}\mathrm{O}_{3})_{n}]{$^{2-}$} & $0,78^{\mathrm{a}}$ & & & & & & Smith y Martel (1976) \\
\hline & 2,06 & & & & & & Irving y Williams (1953) \\
\hline \multirow{3}{*}[\mathrm{Ni}(\mathrm{SO}_{4})_{n}]{$^{2-}$} & $0,57^{\mathrm{a}}$ & & & & & & Smith y Martel (1976) \\
\hline & 2,40 & & & & & & Irving y Williams (1953) \\
\hline & 2,44 & $1,42^{\mathrm{a}}$ & & & & & Buffle et al. (2007) \\
\hline
\end{tabular}

${ }^{\mathrm{a}}$ Fuerza iónica $(\mathrm{I})=0,5 \mathrm{y}$ para el resto $\mathrm{I}=1$

$\left[\mathrm{Ni}^{2+}\right]=\min \left[\mathrm{Ni}^{2+}\right]=\min \left\{\begin{array}{l}\mathrm{Kps}_{\left[\mathrm{NiCO}_{3}\right]} \cdot\left[\mathrm{CO}_{3}^{2-}\right]^{-1} ; \\ \mathrm{Kp}_{\left.[\mathrm{Ni} \mathrm{OH})_{2}\right]} \cdot 10^{28-2 \mathrm{pH}}\end{array}\right\}$

A partir del balance de dióxido de carbono Ec. (22), la concentración del ión carbonato, a $25^{\circ} \mathrm{C}$, se expresa Ec. (23) por:

$$
\begin{gathered}
\mathrm{CO}_{2(a c)}=\left[\mathrm{CO}_{3}^{2-}\right]+\left[\mathrm{HCO}_{3}^{-}\right]+\left[\mathrm{H}_{2} \mathrm{CO}_{3}\right]+\left[\mathrm{H}_{2} \mathrm{NCOO}^{-}\right] \\
{\left[\mathrm{CO}_{3}^{2-}\right]=\frac{\left[\mathrm{CO}_{2}\right]_{T}}{1+10^{p k_{2}-p H}+10^{p k_{1}+p k_{2}-2 p H}}} \\
{\left[\mathrm{CO}_{3}^{2-}\right]=\frac{\left[\mathrm{CO}_{2}\right]_{T}}{1+10^{10,33-p H}+10^{16,68-2 p H}}}
\end{gathered}
$$

La constante de estabilidad de los compuestos de coordinación de níquel con ligando $\left[\mathrm{NH}_{3}\right]$ se plantea por la Ec. (24):

$$
\begin{aligned}
& {\left[\mathrm{Ni}^{2+}\right]+n \mathrm{NH}_{3} \leftrightarrow \mathrm{Ni}\left(\mathrm{NH}_{3}\right)_{n}^{2+} \mathrm{n}=1,2 \ldots, 6} \\
& \beta=\frac{\mathrm{Ni}\left(\mathrm{NH}_{3}\right)_{n}^{2+}}{\left[\mathrm{Ni}^{2+}\right] \cdot\left[\mathrm{NH}_{3}\right]^{n}} \\
& {\left[\mathrm{Ni}\left(\mathrm{NH}_{3}\right)_{n}^{2+}\right]_{T}=\left[\mathrm{Ni}^{2+}\right] \cdot\left\{\begin{array}{l}
10^{2,81} \cdot\left[\mathrm{NH}_{3}\right]+10^{5,04} \cdot \\
{\left[\mathrm{NH}_{3}\right]^{2}+10^{6,77} \cdot} \\
{\left[\mathrm{NH}_{3}\right]^{3}+10^{7,96} \cdot\left[\mathrm{NH}_{3}\right]^{4}} \\
+10^{8,71} \cdot\left[\mathrm{NH}_{3}\right]^{5}+ \\
10^{8,74} \cdot\left[\mathrm{NH}_{3}\right]^{6}
\end{array}\right\}}
\end{aligned}
$$

Para ligando tiosulfato $\left[\mathrm{S}_{2} \mathrm{O}_{3}{ }^{2-}\right]$ y sulfato $\left[\mathrm{SO}_{4}{ }^{2-}\right]$ Ecs. (25) y (26):

$$
\begin{gathered}
{\left[\mathrm{Ni}\left(\mathrm{S}_{2} \mathrm{O}_{3}\right)_{2}\right]^{2-}=\left[\mathrm{Ni}^{2+}\right] \cdot 10^{2,06} \cdot\left[\mathrm{S}_{2} \mathrm{O}_{3}\right]^{2}} \\
{\left[\mathrm{Ni}\left(\mathrm{SO}_{4}\right)_{2}\right]^{2-}=\left[\mathrm{Ni}^{2+}\right] \cdot 10^{2,4} \cdot\left[\mathrm{SO}_{4}\right]^{2}}
\end{gathered}
$$

El balance de hidróxido de níquel, por las Ecs. (27) y (28):

$$
\begin{aligned}
& {[\mathrm{Ni}(\mathrm{OH})]_{T}=} {\left[\mathrm{Ni}(\mathrm{OH})^{+}\right]+\left[\mathrm{Ni}(\mathrm{OH})_{2(a c)}\right] } \\
&+\left[\mathrm{Ni}(\mathrm{OH})_{3_{(a c)}}\right] \\
& {[\mathrm{Ni}(\mathrm{OH})]_{T}=\left[\mathrm{Ni} i^{2+}\right] \cdot\left\{\begin{array}{l}
10^{p H-9,03}+10^{2 p H-19,45} \\
+10^{3 p H-30,67}
\end{array}\right\} }
\end{aligned}
$$

El níquel total está en la forma de níquel libre, en compuesto de coordinación e hidroxilado, Ecs. (29) y (30):

$$
\begin{aligned}
{[\mathrm{Ni}]_{T}=} & {\left[\mathrm{Ni}{ }^{2+}\right]+\left[\mathrm{Ni}\left(\mathrm{NH}_{3}\right)\right]_{T}+[\mathrm{Ni}(\mathrm{OH})]_{T}+} \\
{\left[\mathrm{Ni}\left(\mathrm{S}_{2} \mathrm{O}_{3}\right)\right]_{T}+\left[\mathrm{Ni}\left(\mathrm{SO}_{4}\right)\right]_{T} } & {\left[\begin{array}{l}
1+10^{2,81} \cdot\left[\mathrm{NH}_{3}\right]+10^{5,08} \cdot\left[\mathrm{NH}_{3}\right]^{2}+ \\
10^{6,85} \cdot\left[\mathrm{NH}_{3}\right]^{3}+10^{8,12} \cdot\left[\mathrm{NH}_{3}\right]^{4} \\
+10^{8,93} \cdot\left[\mathrm{NH}_{3}\right]^{5}+10^{9,08} \cdot\left[\mathrm{NH}_{3}\right]^{6}+ \\
10^{p H-9,03}+10^{2 p H-19,45}+10^{3 p H-30,67} \\
+10^{2,06} \cdot\left[\mathrm{S}_{2} \mathrm{O}_{3}\right]^{2}+10^{2,40} \cdot\left[\mathrm{SO}_{4}\right]^{2}
\end{array}\right\} }
\end{aligned}
$$


El amoníaco total será, Ecs. $(31,32)$ :

$$
\begin{aligned}
& {\left[\mathrm{NH}_{3}\right]_{T}=} {\left[\mathrm{NH}_{3}\right]+\left[\mathrm{NH}_{4}^{+}\right]+\left[\mathrm{H}_{2} \mathrm{NCOO}^{-}\right]+} \\
& \sum \beta \cdot\left[\mathrm{NH}_{3}\right]^{n} \\
& {\left[\mathrm{NH}_{3}\right]_{T}=\left[\mathrm{NH}_{3}\right]+\left\{\begin{array}{l}
1+10^{p K a-p H}+\left[\mathrm{CO}_{3}^{2-}\right] \cdot 10^{10,78-p H} \\
+10^{2,81} \cdot\left[\mathrm{Ni}^{2+}\right]+10^{5,04} . \\
{\left[\mathrm{Ni}^{2+}\right] \cdot\left[\mathrm{NH}_{3}\right]+10^{6,77} .} \\
{\left[\mathrm{Ni}^{2+}\right] \cdot\left[\mathrm{NH}_{3}\right]^{2}+10^{7,96} .} \\
{\left[\mathrm{Ni}^{2+}\right] \cdot\left[\mathrm{NH}_{3}\right]^{3}+10^{8,71} .} \\
{\left[\mathrm{Ni}^{2+}\right] \cdot\left[\mathrm{NH}_{3}\right]^{4}+10^{8,74} .} \\
{\left[\mathrm{Ni}^{2+}\right] \cdot\left[\mathrm{NH}_{3}\right]^{5}}
\end{array}\right.}
\end{aligned}
$$

Se calculó la concentración de níquel (II) disuelto y de carbonato por las Ecs. (21) y (23), respectivamente. Aplicando el método numérico de Newton Rapson, se estimó el amoníaco libre y el amoníaco total por Ec. (32). Finalmente, se calculó el Ni(II) por Ec. (30). Este algoritmo se desarrolló en una aplicación de software y se exportaron los datos al Microsoft Excel.

\section{RESULTADOS Y DISCUSIÓN}

\subsection{Análisis del modelo de níquel disuelto para el proceso en Nicaro}

Se obtuvo un modelo estadístico para el pronóstico de la concentración $\left(\mathrm{g} \cdot \mathrm{L}^{-1}\right)$ de níquel disuelto en la suspensión de $\mathrm{CBN}$, en función del $\mathrm{pH}$ de la suspensión; y de la concentración de azufre (S), amoníaco $\left(\mathrm{NH}_{3}\right)$ y dióxido de carbono $\left(\mathrm{CO}_{2}\right)$ en el Licor Producto que alimenta a las columnas de destilación. La temperatura en el tope de la columna y el flujo de operación se mantuvieron en un rango estrecho y no ejercieron significación estadística sobre la concentración de Ni disuelto, Ec. (33):

$$
\begin{aligned}
\mathrm{Ni}_{d}= & a_{1}+a_{2} \cdot p H+a_{3} \cdot p H^{2}+a_{4} \cdot\left[\frac{\mathrm{CO}_{2}}{\mathrm{~S}}\right] \\
& +a_{5} \cdot\left[\frac{\mathrm{NH}}{\mathrm{CO}_{2}}\right] \cdot\left[\frac{\mathrm{Ni}}{\mathrm{S}}\right] \cdot p H+a_{6} \cdot\left[\frac{\mathrm{NH}}{\mathrm{CO}_{2}}\right] \cdot p H \\
& +a_{7} \cdot\left[\frac{\mathrm{NH}}{\mathrm{CO}_{2}}\right] \cdot\left[\frac{\mathrm{Ni}}{\mathrm{S}}\right] \cdot p H
\end{aligned}
$$

Las constantes del modelo para el proceso en Nicaro $(\mathrm{N})$, se muestran en la Tabla 3, válidos en los rangos: $8,0<\mathrm{pH}<9,0 ; 6,5<\mathrm{CO}_{2} / \mathrm{S}<10,2 ; 1,6$ $<\mathrm{NH}_{3} / \mathrm{CO}_{2}<1,9 ; 1,0<\mathrm{Ni} / \mathrm{S}<2,0$. Se emplearon 76 muestras.

El valor-p en la tabla ANOVA resultó inferior a 0,01 , demostrando una relación estadísticamente significativa entre las variables para un nivel de confianza del 99\%. El modelo explica el 95,6\% de la variabilidad en el níquel disuelto $\left(\mathrm{R}^{2}\right)$, con un error estándar de la estimación de 0,029 y error absoluto medio de 0,023. La prueba de Durbin-Watson (DW) fue positiva, igual a 2,02 $(\mathrm{P}=0,33)$, indicando que no hay correlación serial significativa. El estadígrafo $\mathrm{R}^{2}$ ajustado por los grados de libertad fue de $95,2 \%$.

La evaluación del modelo $(\mathrm{N})$ indica que cuando se opera con alta relación Ni/S en el Licor Producto, se obtiene en la suspensión de $\mathrm{CBN}$ menor concentración de níquel disuelto y por tanto, menor gasto en el proceso posterior de precipitación química (Fig. 1a) (Rojas-Vargas y Trujillo, 2012; Magaña Haynes y Rojas-Vargas, 2013).

El azufre se incorpora al proceso fundamentalmente, por contaminación del mineral durante el secado con los gases de combustión del petróleo, y con el petróleo aditivo suministrado a los hornos de reducción. Este compuesto debe mantenerse al mínimo al ejercer un papel fundamental en el incremento de la concentración de níquel disuelto, luego, la relación $\mathrm{CO}_{2} / \mathrm{S}$ también debe ser máxima.

En el proceso de lixiviación se recomienda operar a una relación $\mathrm{NH}_{3} / \mathrm{CO}_{2}$ entre 1,60 y 1,75 para favorecer las extracciones de níquel y cobalto; una correcta carbonatación también beneficia la destilación, puesto que propicia la precipitación del CBN y se obtiene menor concentración de níquel disuelto (ver Fig. 1b), (Chang y Rojas-Vargas, 2009).

En la medida que la alcalinidad de la suspensión disminuye a $\mathrm{pH}$ inferior a 9 , hay una tendencia

TABLA 3. Constantes ${ }^{\mathrm{a}}$ del modelo de pronóstico de níquel disuelto.

\begin{tabular}{lccccccc}
\hline Plantas & $\boldsymbol{a}_{1}$ & $\boldsymbol{a}_{2}$ & $\boldsymbol{a}_{3}$ & $\boldsymbol{a}_{4}$ & $\boldsymbol{a}_{5}$ & $\boldsymbol{a}_{6}$ & $\boldsymbol{a}_{7}$ \\
\hline $\mathrm{N}^{\mathrm{b}}$ & 29,3372 & $-6,71973$ & 0,39215 & $-0,01492$ & 0,78878 & 0,02686 & $-0,1073$ \\
$\mathrm{PG}^{\mathrm{c}}$ & 30,1204 & $-6,84078$ & 0,39038 & 0 & 0 & 0 & 0 \\
\hline
\end{tabular}

${ }^{\mathrm{a}} a_{\mathrm{n}}$ Constante del modelo con $\mathrm{n}=1$ a $7,{ }^{\mathrm{b}}$ Nicaro, ${ }^{\mathrm{c}}$ Punta Gorda 

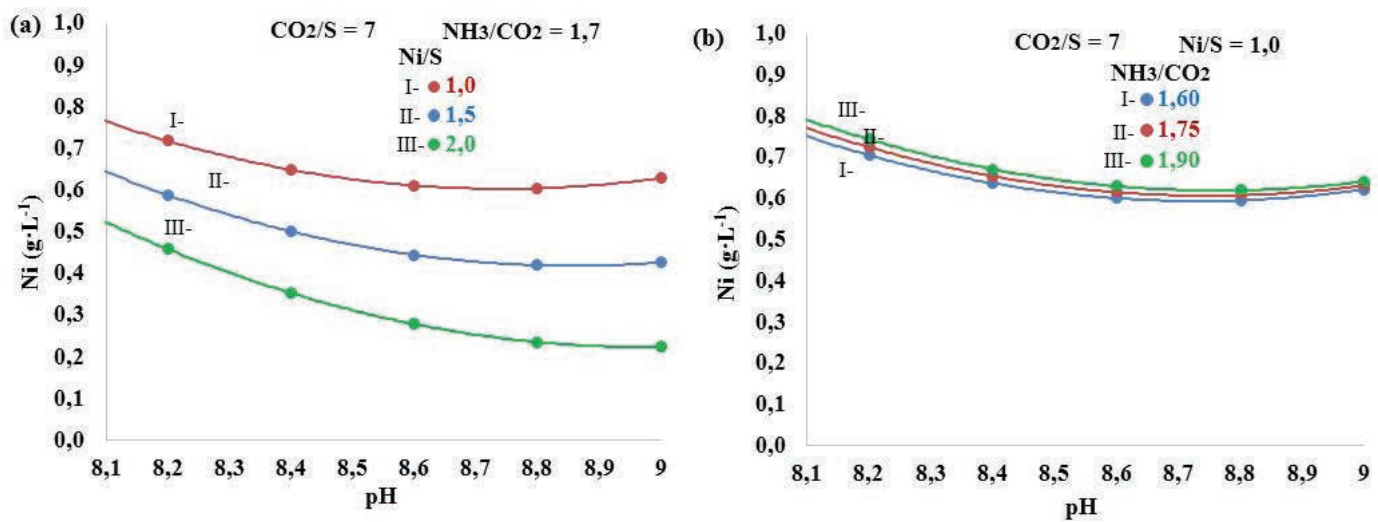

Figura 1. Evaluación del modelo de pronóstico de níquel disuelto para Nicaro: a) Variación Ni/S, y b) Variación $\mathrm{NH}_{3} / \mathrm{CO}_{2}$.

al incremento de la concentración de níquel disuelto, lo que se ha denominado "sobre destilación". (RojasVargas y Trujillo, 2012; Magaña Haynes y RojasVargas, 2013).

El dióxido de carbono, azufre y el amoníaco en disolución acuosa pueden formar compuestos complejos de níquel, incidiendo en la lixiviación del níquel contenido en el CBN. En la Fig. 2, se muestran los valores observados y predichos de níquel disuelto, para dos grupos de muestras obtenidas en la planta productora en Nicaro.

\subsection{Modelo de níquel disuelto para el proceso en Punta Gorda}

Las constantes del modelo de pronóstico para la planta productora en Punta Gorda (PG) se muestran en la Tabla 3, válidos en los rangos: $7,4<\mathrm{pH}$ $<9,0 ; 10,4<\mathrm{CO}_{2} / \mathrm{S}<13,8 ; 1,5<\mathrm{NH}_{3} / \mathrm{CO}_{2}<2,0$; $1,8<\mathrm{Ni} / \mathrm{S}<3,2$. Se emplearon 43 muestras.
El modelo explica el 94,7\% de la variabilidad en el níquel disuelto $\left(\mathrm{R}^{2}\right)$, con un error estándar de 0,05 y error absoluto medio de 0,038 . La prueba DW fue igual a $1,5(\mathrm{P}=0,08)$ y el estadígrafo $\mathrm{R}^{2}$ ajustado por los grados de libertad de $94,5 \%$.

La concentración de níquel disuelto mostró una dependencia polinomial de segundo orden del $\mathrm{pH}$ de la suspensión de CBN. La operación de las columnas en PG, se realiza alcanzando valores de $\mathrm{pH}$ próximos a neutro, por lo que se debe considerar esta variable en el método de control para disminuir el $\left[\mathrm{Ni}^{2+}\right]$ en disolución.

En esta planta se alimenta solución amoniacal con alto $\mathrm{CO}_{2}$ por un plato lateral de las columnas, lo que favorece las propiedades físico - químicas del CBN; la concentración de azufre en el Licor Producto procesado en PG fue inferior al procesado en Nicaro y la relación $\mathrm{Ni} / \mathrm{S}$ resultó más favorable; en consecuencia, las variables concentración de $\mathrm{NH}_{3}, \mathrm{CO}_{2}, \mathrm{~S}$ y sus proporciones, no
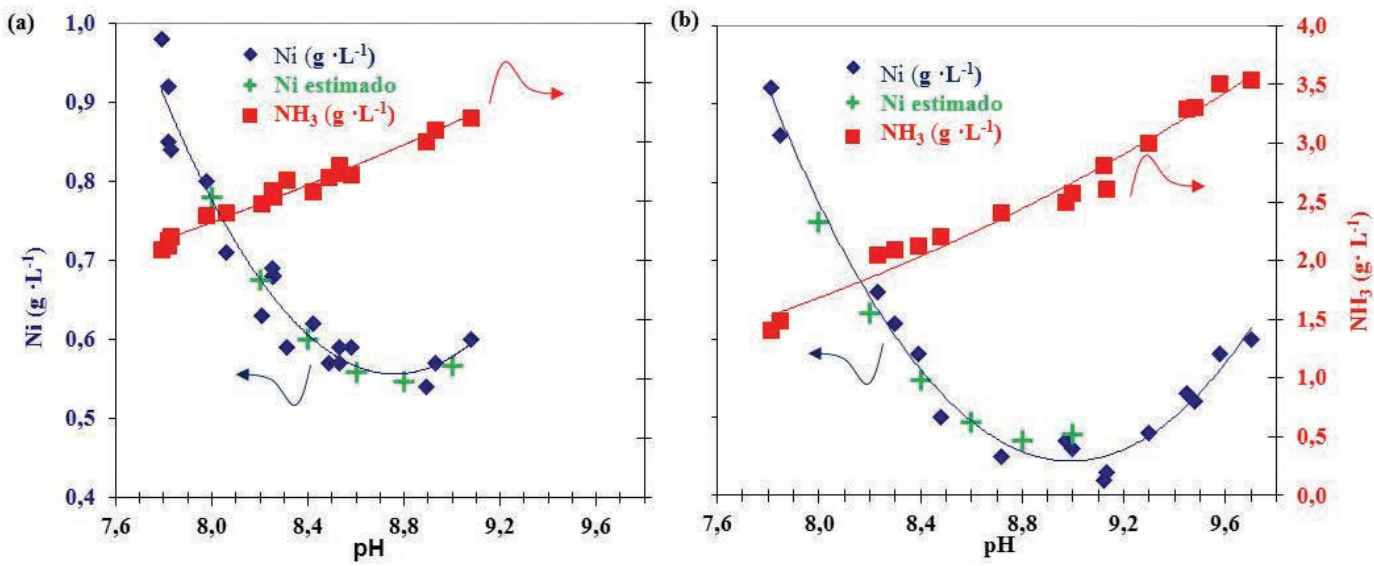

Figura 2. Validación del modelo de níquel disuelto para Nicaro: a) $\left[\mathrm{NH}_{3} / \mathrm{CO}_{2}\right]=1,62[\mathrm{Ni} / \mathrm{S}]=1,16\left[\mathrm{CO}_{2} / \mathrm{S}\right]=7,7 \mathrm{y}$ b) $\left[\mathrm{NH}_{3} / \mathrm{CO}_{2}\right]=1,79[\mathrm{Ni} / \mathrm{S}]=1,33\left[\mathrm{CO}_{2} / \mathrm{S}\right]=7,0$. 
ejercieron significación estadísticamente significativa sobre el níquel disuelto.

\subsection{Simulación del $\mathrm{Ni}$ disuelto en el sistema $\mathrm{Ni}(\mathrm{II})-\mathrm{NH}_{3}-\mathrm{CO}_{3}{ }^{2-}-\mathrm{S}_{2} \mathrm{O}_{3}{ }^{2-}-\mathrm{SO}_{4}{ }^{2-}-\mathrm{H}_{2} \mathrm{O}$}

Aplicando las Ecs. (21) a (32), se simuló la solución que acompaña el $\mathrm{CBN}$ en el sistema $\mathrm{Ni}$ (II) $-\mathrm{NH}_{3}-\mathrm{CO}_{3}{ }^{2-}-\mathrm{S}_{2} \mathrm{O}_{3}{ }^{2-}-\mathrm{SO}_{4}{ }^{2-}-\mathrm{H}_{2} \mathrm{O}$ y se estimó la concentración del níquel disuelto a diferentes valores de $\mathrm{pH}$, concentración de $\left[\mathrm{CO}_{2}\right],\left[\mathrm{NH}_{3}\right]$ e iones $\left[\mathrm{S}_{\mathrm{x}} \mathrm{O}_{\mathrm{y}} \mathrm{z}^{-}\right]$(Fig. 3).

Cuando aumenta la concentración de amoníaco de 0,01 a $0,5 \mathrm{~mol} \cdot \mathrm{L}^{-1}$ (ver Fig. 3a), aumenta el $\left[\mathrm{Ni}^{2+}\right]$ y el rango de estabilidad de las especies químicas formadas. A pH $>8$, la concentración de $\left[\mathrm{NH}_{3}\right]$ libre crece y se forman compuestos de coordinación, aumentando el $\left[\mathrm{Ni}^{2+}\right]$ para concentración $0,1 \mathrm{M}$ y $0,5 \mathrm{M}$ de $\left[\mathrm{NH}_{3}\right]_{\mathrm{T}}$. A pH>10, el $\left[\mathrm{NH}_{4}{ }^{+}\right]$disminuye, y el níquel precipita como $\mathrm{Ni}(\mathrm{OH})_{2}$ y CBN (Guo et al., 2004).
A mayor concentración de dióxido de carbono (ver Fig. 3b), menor será la concentración de $\left[\mathrm{Ni}^{2+}\right]$, lo que se evidencia en la práctica industrial a través del modelo estadístico Ec. (33), al operar con Licor Producto correctamente carbonatadas y alimentar solución amoniacal con alto $\mathrm{CO}_{2}$ por un plato lateral. Esto se debe al decrecimiento del amoníaco libre y a la formación del CBN cuya molécula posee menos $\left[\mathrm{OH}^{-}\right]$y $\left[\mathrm{SO}_{4}{ }^{2-}\right]$. Resultados experimentales en una minicolumna de destilación refieren semejante comportamiento (Rojas-Vargas y Trujillo, 2012; Magaña Haynes, 2016).

$\mathrm{Al}$ aumentar la concentración de iones $\left[\mathrm{S}_{\mathrm{x}} \mathrm{O}_{\mathrm{y}}{ }^{\mathrm{z}}\right]$ y por tanto de azufre (ver Fig. 3c), se incrementa el $\left[\mathrm{Ni}^{2+}\right]$ y el mínimo de concentración se desplaza hacia un $\mathrm{pH}$ más alcalino en el intervalo de $7,5<\mathrm{pH}<8,5$, donde se aprecia una parábola bien definida tal como la tendencia lograda con los valores experimentales del proceso industrial (RojasVargas y Trujillo, 2012), (Fig. 2).
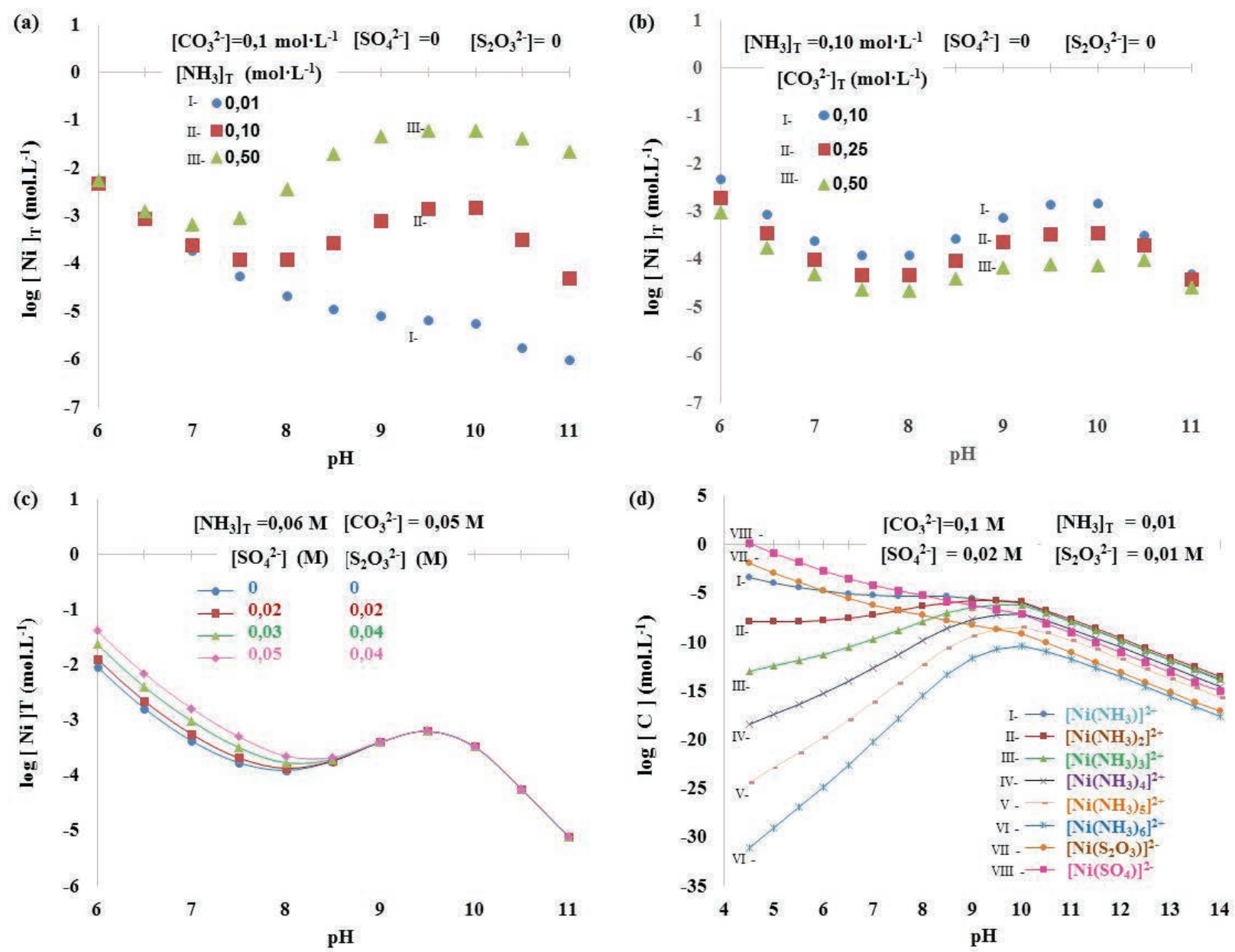

FIGURA 3. Concentración de níquel disuelto en el sistema $\mathrm{Ni}(\mathrm{II})-\mathrm{NH}_{3}-\mathrm{CO}_{3}{ }^{2-}-\mathrm{S}_{2} \mathrm{O}_{3}{ }^{2-}-\mathrm{SO}_{4}{ }^{2-}-\mathrm{H}_{2} \mathrm{O}$ : a) Variación de $\left[\mathrm{NH}_{3}\right]_{\mathrm{T}}\left(\mathrm{mol} \cdot \cdot^{-1}\right)$; b) Variación de $\left[\mathrm{CO}_{3}^{2-}\right]_{\mathrm{T}}\left(\mathrm{mol} \cdot \mathrm{l}^{-1}\right)$; c) Variación de $\left[\mathrm{S}_{\mathrm{x}} \mathrm{O}_{\mathrm{y}}{ }^{\mathrm{l}}\right]\left(\mathrm{mol} \cdot \mathrm{l}^{-1}\right)$; y d) Especies coordinadas de níquel (II) $\left(\mathrm{mol} \cdot \mathrm{L}^{-1}\right)$. 
En el rango de $7,5<\mathrm{pH}<8,5$, se determinaron las constantes de los polinomios de $\left[\mathrm{Ni}^{2+}\right]$, y se pudo apreciar que cuando incrementa la concentración de iones $\left[\mathrm{S}_{\mathrm{x}} \mathrm{O}_{\mathrm{y}}{ }^{\mathrm{z}-}\right]$ disminuye la abertura de la parábola, determinada por el coeficiente cuadrático del polinomio e igual sucede, al disminuir la concentración de $\left[\mathrm{CO}_{3}{ }^{2-}\right]$ lo que significa que con menor variación del $\mathrm{pH}$, la variación del $\left[\mathrm{Ni}^{2+}\right]$ será superior. En la medida que disminuye el $\mathrm{pH}$, predomina la concentración de especies de níquel coordinadas con los iones $\left[\mathrm{S}_{\mathrm{x}} \mathrm{O}_{\mathrm{y}}{ }^{\mathrm{z}}\right]$, lo que ratifica la necesidad de operar con baja concentración de azufre en las soluciones alimentadas a la destilación, para reducir los gastos de precipitación (Fig. 3d).

\subsection{Lixiviación del níquel del CBN}

$\mathrm{La}$ "sobre destilación" del CBN que sucede en las columnas del proceso carbonato amoniacal, en el sistema $\mathrm{Ni}$ (II)- $\mathrm{NH}_{3}-\mathrm{CO}_{3}{ }^{2-}-\mathrm{S}_{2} \mathrm{O}_{3}{ }^{2-}-\mathrm{SO}_{4}{ }^{2-}-\mathrm{H}_{2} \mathrm{O}$, se puede explicar por la lixiviación del níquel de la mezcla de compuestos: carbonato de níquel $\left[\mathrm{NiCO}_{3}\right]$, sulfato de níquel $\left[\mathrm{NiSO}_{4}\right]$, hidróxido de níquel $\left[\mathrm{Ni}(\mathrm{OH})_{2}\right]$ y oxido de níquel $[\mathrm{NiO}]$ formando sales y compuestos de coordinación (Ver Fig. 4).

La fracción molar de iones presentes en el sistema en equilibrio en función del $\mathrm{pH}$ se representa en la Fig. 5. Puede apreciarse que a $\mathrm{pH}>8,2$ se forma el anión carbonato $\left[\mathrm{CO}_{3}{ }^{2-}\right]$ con un $1 \%$ molar y alcanza un $14,6 \% \mathrm{M}$ a $\mathrm{pH}=9,6$. Por su parte, el amoníaco libre tiene un $5 \%$ a $\mathrm{pH}=8,0$ y comienza a incrementar hacia $\mathrm{pH}$ alcalino, en cambio el catión amonio disminuye su fracción molar y a un $\mathrm{pH}=10,5$ representa un 5\%. A pH=9,24 el cociente entre la fracción de iones amonio y amoníaco libre es igual a $1\left[\mathrm{NH}_{4}{ }^{+}\right]=\left[\mathrm{NH}_{3}\right]$. En el intervalo de $8,2<\mathrm{pH}<10,5$ la presencia de $\left[\mathrm{NH}_{3}\right],\left[\mathrm{NH}_{4}{ }^{+}\right]$y $\left[\mathrm{CO}_{3}{ }^{2-}\right]$ propician la formación de complejos carbonato ammina - níquel (II), tal como se representó en la Fig. 3 (a, b, d), Ec. (34):

$$
\mathrm{NiCO}_{3}+2 \mathrm{NH}_{3}+3 \mathrm{NH}_{4}^{+} \rightarrow \mathrm{Ni}\left(\mathrm{NH}_{3}\right)_{6} \mathrm{CO}_{3}
$$

El diagrama Eh-pH (Fig. 6a), sistema $\mathrm{Ni}-\mathrm{NH}_{3}-$ $\mathrm{S}_{2} \mathrm{O}_{3}{ }^{2-}-\mathrm{H}_{2} \mathrm{O}$, simula la concentración de iones en condiciones de "sobre destilación", a $25^{\circ} \mathrm{C},\left[\mathrm{Ni}^{2+}\right]=$ $0,02 \mathrm{M} ;\left[\mathrm{NH}_{4} \mathrm{OH}\right]=0,1 \mathrm{M} ;\left[\mathrm{S}_{2} \mathrm{O}_{3}{ }^{2-}\right]=0,03 \mathrm{M}$. Dicho diagrama aporta información útil, puesto que representa áreas de estabilidad termodinámica de las especies sólidas y acuosas en función del potencial (Eh) y del pH. A las condiciones de "sobre destilación", el área de estabilidad de la especie hexammina níquel (II) $\left[\mathrm{Ni}\left(\mathrm{NH}_{3}\right)_{6}\right]^{2+}$ es pequeña debido a la baja concentración de amoníaco, en consonancia con los resultados mostrados en la Fig. 3d; además, es posible la precipitación de óxidos de níquel y la formación del anión $\left[\mathrm{Ni}\left(\mathrm{S}_{2} \mathrm{O}_{3}\right)_{2}\right]^{2-}$ en un amplio rango de potencial y estabilidad a $\mathrm{pH}$ inferior a 7,2 (Arima et al., 2004).

Los iones $\left[\mathrm{S}_{\mathrm{x}} \mathrm{O}_{\mathrm{y}} \mathrm{z}^{-}\right]$provienen del proceso de lixiviación, donde el azufre se oxida a tiosulfato $\left[\mathrm{S}_{2} \mathrm{O}_{3}{ }^{2-}\right]$, politionatos $\left(\mathrm{S}_{\mathrm{n}} \mathrm{O}_{\mathrm{m}}{ }^{2-}, 2<\mathrm{n}<6\right)$, sulfito $\left[\mathrm{SO}_{3}{ }^{2-}\right]$, sulfato $\left[\mathrm{SO}_{4}{ }^{2-}\right]$ y sulfamato $\left[\mathrm{SO}_{3} \mathrm{NH}_{2}{ }^{-}\right]$, (Grosse et al., 2003; $\mathrm{Xu}$ et al., 2017), Ec. (35):

$$
\begin{aligned}
& \mathrm{S}^{2-} \rightarrow \mathrm{S}^{0} \rightarrow \mathrm{S}_{2} \mathrm{O}_{3}^{2-} \leftrightarrow \mathrm{S}_{4} \mathrm{O}_{6}^{2-} \rightarrow \mathrm{S}_{3} \mathrm{O}_{6}^{2-} \rightarrow \mathrm{SO}_{3}^{2-} \\
& \rightarrow \mathrm{SO}_{4}^{2-}+\mathrm{SO}_{3} \mathrm{NH}_{2}^{-}
\end{aligned}
$$

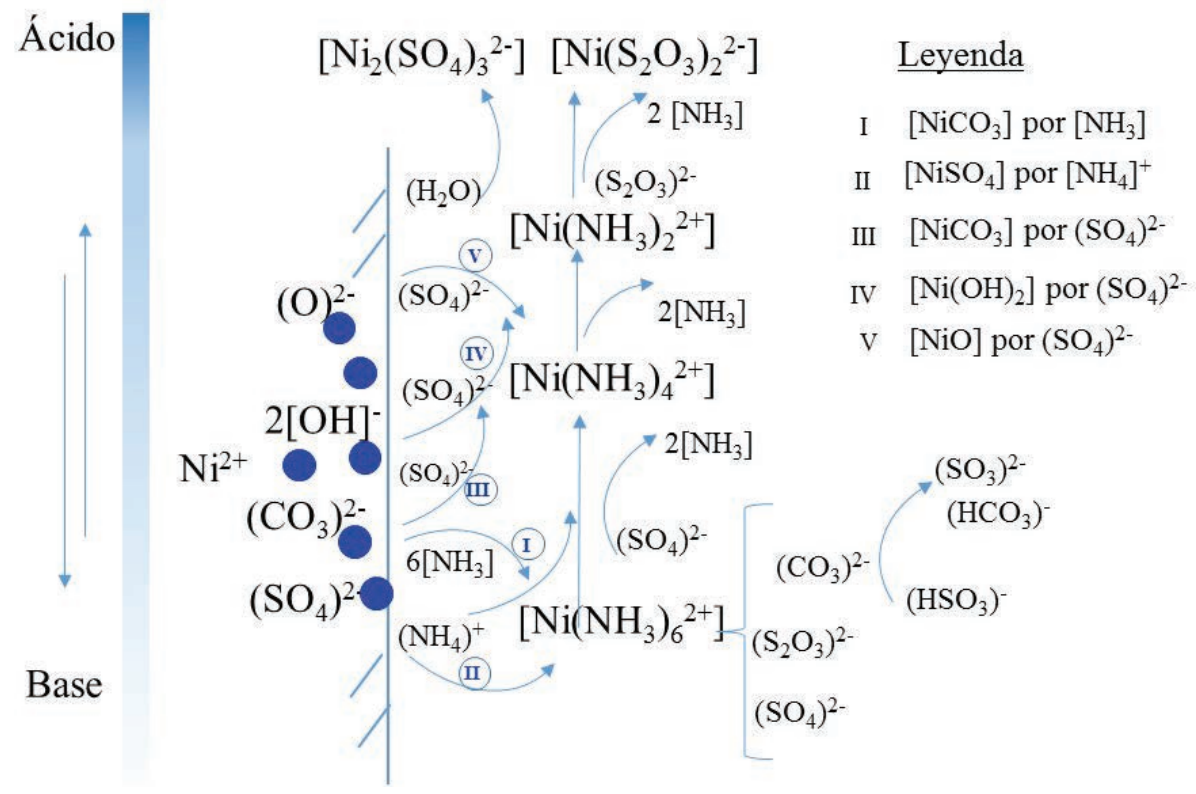

Figura 4. Lixiviación del carbonato básico de níquel en el sistema $\mathrm{Ni}$ (II)- $-\mathrm{NH}_{3}-\mathrm{CO}_{3}{ }^{2-}-\mathrm{S}_{2} \mathrm{O}_{3}{ }^{2-}-\mathrm{SO}_{4}{ }^{2-}-\mathrm{H}_{2} \mathrm{O}$. 


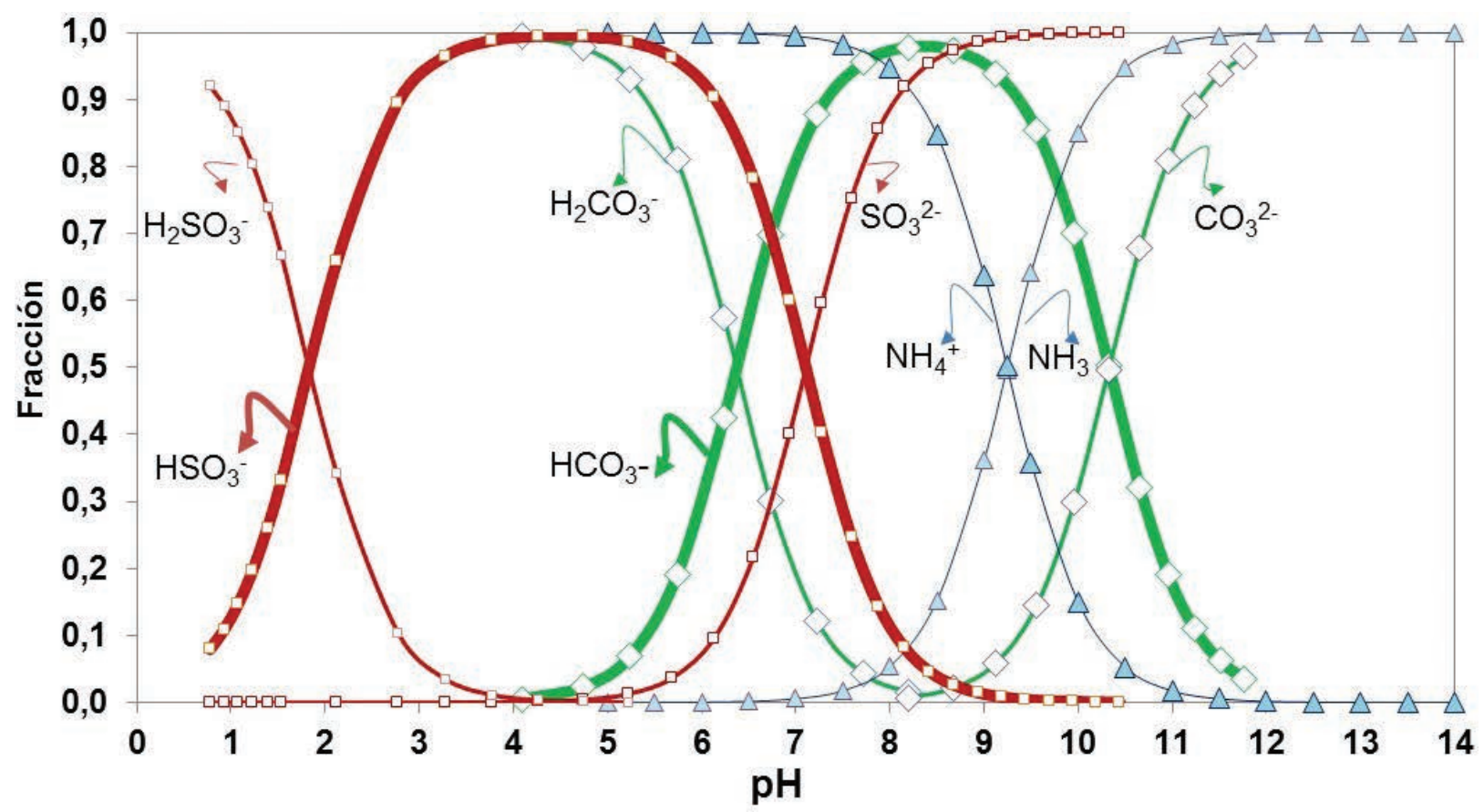

Figura 5. Especies químicas en el sistema $\mathrm{NH}_{3}-\mathrm{CO}_{2}-\mathrm{SO}_{2}-\mathrm{H}_{2} \mathrm{O}$.
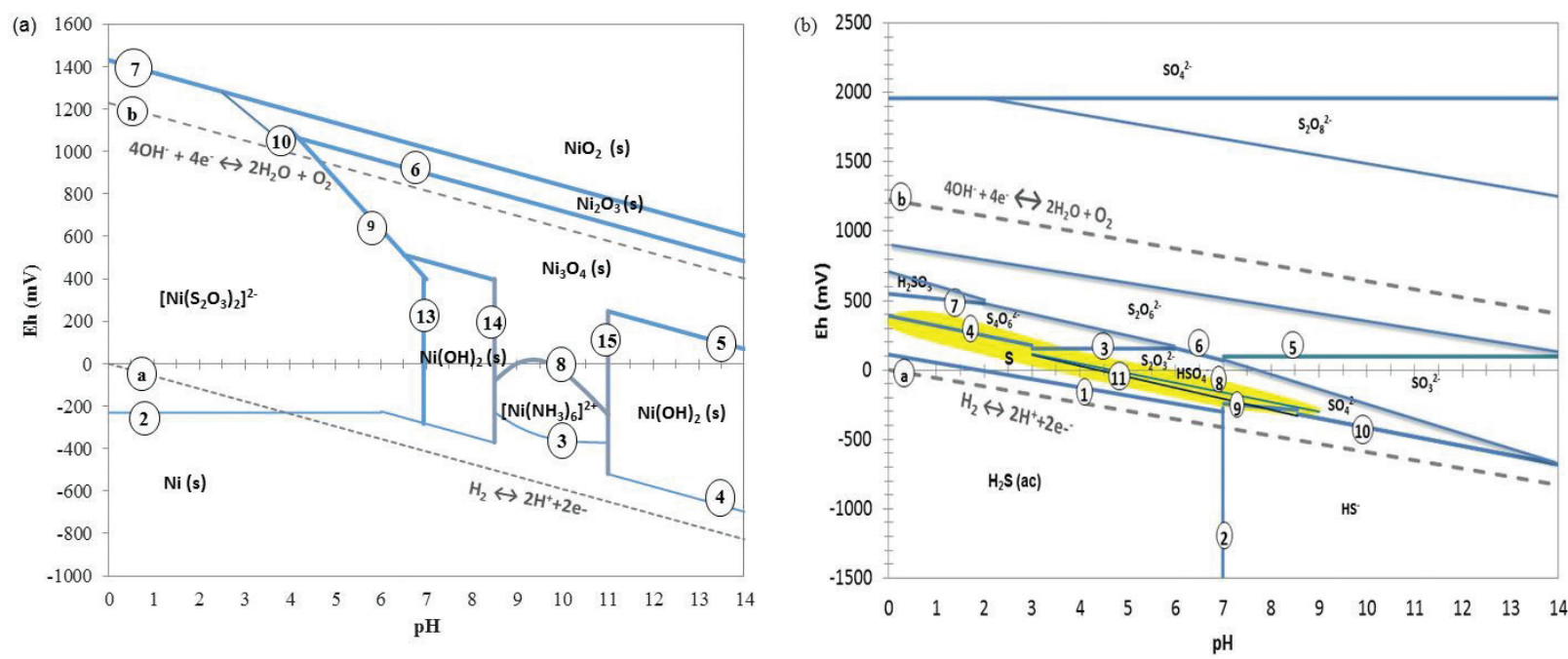

FIgURA 6. Diagrama Eh-pH: a) Sistema Ni- $\mathrm{NH}_{3}-\mathrm{S}_{2} \mathrm{O}_{3}{ }^{2-}-\mathrm{H}_{2} \mathrm{O}$ a $25^{\circ} \mathrm{C},\left[\mathrm{Ni}^{2+}\right]=0,02 \mathrm{M} ;\left[\mathrm{NH}_{4} \mathrm{OH}\right]=0,1 \mathrm{M} ;\left[\mathrm{S}_{2} \mathrm{O}_{3}{ }^{2-}\right]=0,03 \mathrm{M}$; $\mathrm{y}$ b) Sistema S- $\mathrm{H}_{2} \mathrm{O}$ a $25^{\circ} \mathrm{C}$, concentración de iones $10^{-1} \mathrm{M}$.

En el diagrama Eh-pH para el sistema $\mathrm{S}-\mathrm{H}_{2} \mathrm{O}$, se aprecian las especies de azufre en disolución acuosa: tiosulfato $\left[\mathrm{S}_{2} \mathrm{O}_{3}{ }^{2-}\right]$, ditiosulfato $\left[\mathrm{S}_{4} \mathrm{O}_{6}{ }^{2-}\right]$, ditionato $\left[\mathrm{S}_{2} \mathrm{O}_{6}{ }^{2-}\right]$, peroxodisulfato $\left[\mathrm{S}_{2} \mathrm{O}_{8}{ }^{2-}\right]$, sulfito $\left[\mathrm{SO}_{3}{ }^{2-}\right]$, sulfato $\left[\mathrm{SO}_{4}{ }^{2-}\right]$ (Fig. 6b). La región sombreada muestra el área de estabilidad termodinámica para el anión tiosulfato $\left[\mathrm{S}_{2} \mathrm{O}_{3}{ }^{2-}\right]$ en el intervalo $0 \leq \mathrm{pH} \leq 9$; coexiste con el anión bisulfato $\left[\mathrm{HSO}_{4}{ }^{-}\right]$en el rango $3,0 \leq \mathrm{pH} \leq 9$ y el sulfato $\left[\mathrm{SO}_{4}{ }^{2-}\right]$ entre $3,0 \leq \mathrm{pH} \leq 14,0$ (Warner, 1988; Aylmore y Muir, 2001).
Luego, considerando la formación de los aniones $\left[\mathrm{Ni}\left(\mathrm{S}_{2} \mathrm{O}_{3}\right)_{2}\right]^{2-}$ y $\left[\mathrm{Ni}\left(\mathrm{S}_{2} \mathrm{O}_{3}\right)\right]^{2-}$ (Fig. 3d y Fig. 6a); y el desplazamiento del $\left[\mathrm{CO}_{3}{ }^{2-}\right.$ ] por el bisulfito $\left[\mathrm{HSO}_{3}{ }^{-}\right]$ por la reacción irreversible reportada por Hikita y Konishi (1981), se puede plantear que los iones $\left[\mathrm{S}_{\mathrm{x}} \mathrm{O}_{\mathrm{y}}{ }^{\mathrm{z}}\right]$ ejercen un papel importante en la lixiviación del níquel del CBN, de ahí la significación estadística del $[\mathrm{S}]$ en el modelo N, Ec. (36):

$$
\mathrm{CO}_{3}^{2-}+\mathrm{HSO}_{3}^{-} \rightarrow \mathrm{SO}_{3}^{2-}+\mathrm{HCO}_{3}^{-}
$$


$\mathrm{El}\left[\mathrm{HSO}_{3}{ }^{-}\right]$posee una fracción molar en disolución desde un $8,2 \%$ a $0,5 \%$ en el rango $8,2 \leq \mathrm{pH} \leq 9,6$ (Fig. 5) por lo que es posible que en este rango de $\mathrm{pH}$ se verifique la reacción Ec. (36) interfiriendo en la formación de los complejos carbonato ammina $\mathrm{Ni}$ (II) por el desplazamiento del anión $\left[\mathrm{CO}_{3}{ }^{2-}\right]$.

Una vez desplazado el $\left[\mathrm{CO}_{3}{ }^{2-}\right]$, aún el catión $\left[\mathrm{Ni}\left(\mathrm{NH}_{3}\right)_{6}\right]^{2+}$ puede estar presente, por combinación con el anión $\left[\mathrm{S}_{2} \mathrm{O}_{3}{ }^{2-}\right]$ formando tiosulfato de hexammina níquel (II) hidratado en el intervalo de $8,5<\mathrm{pH}<11,0$ donde $\mathrm{n}$ alcanza valores de $0,2,3,6$; Ec. (37):

$$
\begin{aligned}
& {\left[\mathrm{Ni}\left(\mathrm{NH}_{3}\right)_{6}\right]^{2+}+\left[\mathrm{S}_{2} \mathrm{O}_{3}\right]^{2-}+n \mathrm{H}_{2} \mathrm{O}} \\
& \rightarrow\left[\mathrm{Ni}\left(\mathrm{NH}_{3}\right)_{6}\right] \mathrm{S}_{2} \mathrm{O}_{3} \cdot n \mathrm{H}_{2} \mathrm{O}
\end{aligned}
$$

En disolución acuosa de sulfato de níquel(II), se han identificado los iones triples del tipo $\left[\mathrm{Ni}_{2}\left(\mathrm{SO}_{4}\right)\right.$ $\left.\left(\mathrm{H}_{2} \mathrm{O}\right)_{\mathrm{n}}\right]^{2+}$ y $\left[\mathrm{Ni}\left(\mathrm{SO}_{4}\right)_{2}\right]^{2-}$. Estos se forman por la respectiva agregación de los iones solvatados $\left[\mathrm{Ni}^{2+}\right]$ y $\left[\mathrm{SO}_{4}{ }^{2-}\right]$ con un par de ión neutral $\left[\mathrm{NiSO}_{4}\right]$, el grado de solvatación del catión $\left[\mathrm{Ni}^{2+}\right]$ controla crucialmente la estructura de los aniones y cationes complejos. También se refieren los iones $\left[\mathrm{Ni}_{3}\left(\mathrm{SO}_{4}\right)_{2}\left(\mathrm{H}_{2} \mathrm{O}\right) \mathrm{n}\right]^{2+}$ $(\mathrm{n}=7-9)$ y $\left[\mathrm{Ni}_{2}\left(\mathrm{SO}_{4}\right)_{3}\right]^{2-}$ (Chen et al., 2005; Schröder et al., 2011).

Luego, la precipitación en las columnas de destilación de un CBN con azufre contenido en su molécula según la Ec. (2), en condiciones de "sobre destilación" puede ser la causa fundamental que propicia la lixiviación del níquel del $\mathrm{CBN}$ en función del $\mathrm{pH}$ de la suspensión. Se plantea que el hidróxido de amonio reacciona con el sulfato de níquel (II) para formar los compuestos complejos de color azul - violeta sulfato de haxammina níquel (II) y el sulfato de tetrammina níquel (II). (Kotelnikova y Mikhailidi, 2012), Ec. (38) y Ec. (39):

$$
\begin{aligned}
& \mathrm{NiSO}_{4}+6 \mathrm{NH}_{4} \mathrm{OH} \rightarrow \mathrm{Ni}\left(\mathrm{NH}_{3}\right)_{6} \mathrm{SO}_{4}+6 \mathrm{H}_{2} \mathrm{O} \\
& \mathrm{NiSO}_{4}+4 \mathrm{NH}_{4} \mathrm{OH} \rightarrow \mathrm{Ni}\left(\mathrm{NH}_{3}\right)_{4} \mathrm{SO}_{4}+4 \mathrm{H}_{2} \mathrm{O}
\end{aligned}
$$

Por su parte, el sulfato de amonio [ $\left.\left(\mathrm{NH}_{4}\right)_{2} \mathrm{SO}_{4}\right]$ también puede estar relacionado con la lixiviación del níquel, tanto a partir del carbonato como del sulfato, hidróxido y el óxido de níquel. En presencia de las especies $\left[\mathrm{NH}_{4}^{+}\right]$y $\left[\mathrm{NH}_{3}\right]$ libre, se forman los compuestos: sulfato de biammina níquel (II), sulfato de tetraammina níquel (II) y la sal doble "Tutton", sulfato de níquel (II) y amonio hexahidratado (Söhnel y Mullin, 1979), Ecs. (40), (41), (42), (43):

$$
\begin{aligned}
& \mathrm{NiCO}_{3}+\left(\mathrm{NH}_{4}\right)_{2} \mathrm{SO}_{4} \rightarrow \mathrm{Ni}\left(\mathrm{NH}_{3}\right)_{4} \mathrm{SO}_{4} \\
& +\mathrm{CO}_{2}+\mathrm{H}_{2} \mathrm{O}
\end{aligned}
$$

$$
\begin{aligned}
& \mathrm{NiSO}_{4}+\left(\mathrm{NH}_{4}\right)_{2} \mathrm{SO}_{4}+6 \mathrm{H}_{2} \mathrm{O} \\
& \rightarrow \mathrm{Ni}\left(\mathrm{NH}_{4}\right)_{2}\left(\mathrm{SO}_{4}\right)_{2} \cdot 6 \mathrm{H}_{2} \mathrm{O} \\
& \mathrm{Ni}(\mathrm{OH})_{2(s)}+\left(\mathrm{NH}_{4}\right)_{2} \mathrm{SO}_{4} \\
& \rightarrow \mathrm{Ni}\left(\mathrm{NH}_{3}\right)_{2} \mathrm{SO}_{4}+2 \mathrm{H}_{2} \mathrm{O} \\
& \mathrm{NiO}+2 \mathrm{NH}_{3}+\left(\mathrm{NH}_{4}\right)_{2} \mathrm{SO}_{4} \\
& \rightarrow \mathrm{Ni}\left(\mathrm{NH}_{3}\right)_{4} \mathrm{SO}_{4}+\mathrm{H}_{2} \mathrm{O}
\end{aligned}
$$

La reacción (42) se aplica en la tecnología de lixiviación sulfato - amoniacal de lateritas $y$ en el proceso de recuperación de níquel de concentrados de hidróxidos de níquel, a $\mathrm{pH}$ entre 6 y 8 . Puede apreciarse que en el diagrama de la Fig. 6a, la presencia del $\mathrm{Ni}(\mathrm{OH})_{2(\mathrm{~s})}$ en los intervalos de 7,2 $\leq \mathrm{pH} \leq 8,5$ y de $11,5 \leq \mathrm{pH} \leq 14,0$ (Kazuyoshi et al., 1974; Jones y Moore, 2001).

Por su parte, la reacción (43) se verifica en procesos de extracción de níquel a partir de nódulos marinos, en presencia de sulfato, tiosulfato y carbonato de amonio, a temperatura de $80^{\circ} \mathrm{C}$ y diámetro promedio inferior a $0,149 \mathrm{~mm}$, en la medida que disminuye el $\mathrm{pH}$, incrementa la lixiviación del metal (Zhang y Cheng, 2007; Biswas et al., 2008).

Cuando el Licor Producto que alimenta a las columnas de destilación posee una relación $\left[\mathrm{NH}_{3}\right]$ $\mathrm{CO}_{2}$ ] inferior a 1,8, al incrementar la concentración de $\left[\mathrm{CO}_{2}\right]$ en el $\mathrm{CBN}$, disminuye el contenido de [S]; este efecto también se logra cuando se alimenta la solución con alto $\mathrm{CO}_{2}$ por un plato lateral, como sucede en PG, aumentando la selectividad hacia el producto deseado $\mathrm{NiCO}_{3}$ con respecto a todo lo que precipitó de níquel disuelto. Si también se opera con baja concentración de azufre en el Licor Producto, tal como se aprecia en la Fig. 3c, determinado por una alta la proporción $[\mathrm{Ni} / \mathrm{S}]$, las reacciones (38) a (43) poseerán baja conversión con significativa dependencia del $\mathrm{pH}$ de la suspensión. Este es el comportamiento que refleja el modelo estadístico PG (Rojas-Vargas y Trujillo, 2012; Magaña Haynes, 2016).

Para reducir el contenido de iones $\left[\mathrm{S}_{\mathrm{x}} \mathrm{O}_{\mathrm{y}}{ }^{\mathrm{z}}\right]$ en el Licor Producto, se debe controlar la aireación en el proceso de lixiviación para evitar una oxidación excesiva del azufre, lo que además resulta favorable para las extracciones de níquel y cobalto. La remoción del azufre disuelto antes de alimentar a destilación también debe ser considerada (Chang y Rojas-Vargas, 2009).

\section{CONCLUSIONES}

- Se obtuvo un modelo estadístico para estimar la concentración de níquel disuelto en la suspensión de carbonato básico de níquel, en función de la concentración de azufre (S), amoníaco 
$\left(\mathrm{NH}_{3}\right)$ y dióxido de carbono $\left(\mathrm{CO}_{2}\right)$ en el Licor Producto que alimenta a las columnas de destilación, y el pH de la suspensión.

- A mayor relación $\mathrm{Ni} / \mathrm{S}, \mathrm{CO}_{2} / \mathrm{S}$ y menor relación $\mathrm{NH}_{3} / \mathrm{CO}_{2}$ en la solución de alimentación, se obtiene en la suspensión de CBN menor concentración de níquel disuelto.

- Cuando la alcalinidad de la suspensión disminuye a $\mathrm{pH}$ inferior a 9, hay una tendencia al incremento de la concentración de níquel disuelto.

\section{AGRADECIMIENTOS}

El autor agradece a los compañeros del laboratorio analítico del Centro de Investigaciones del Níquel en Nicaro.

\section{REFERENCIAS}

Arima, H., Fujita, T, Yen, W.T. (2004). Using Nickel as a Catalyst in Ammonium Thiosulfate Leaching for Gold Extraction. Mater. Trans. 45 (2), 516-526. https://doi.org/ 10.2320/matertrans.45.516.

Aylmore, M.G., Muir, D.M. (2001). Thiosulfate leaching of gold - A review. Miner. Eng. 14 (2), 135-174. https://doi.org/ 10.1016/S0892-6875(00)00172-2.

Biswas, A., Chakraborti, N., Sen, P.K. (2008). Optimization of nonferrous metals recovery from sea nodules in a hydrometallurgical circuit using multi-objetive evolutionary and genetic algorithms. Hydrometallurgy 2008, Proceedings of the Sixth International Symposium, pp. 298-315.

Buffle, J., Zhang, Z., Startchev, K. (2007). Metal flux and dynamic speciation at (bio) interfaces. Part I: Critical evaluation and compilation of physico-chemical parameters for complexes with simple ligands and fulvic/humic substances. Environ. Sci. Technol. 41 (22), 7609-7620. https://doi. org/10.1021/es070702p.

Chang, C.A.R., Rojas-Vargas, A. (2009). La lixiviación del proceso Caron: Síntesis del conocimiento para su perfeccionamiento industrial. Parte II. Tecnología Química 29 (1), $96-105$.

Chen, T., Hefter, G., Buchner, R. (2005). Ion Association and Hydration in Aqueous Solutions of Nickel(II) and Cobalt(II) Sulfate. J. Solution Chem. 34 (9), 1045-1066. https://doi.org/10.1007/s10953-005-6993-5.

Edwards, T.J., Maurer, G., Newman, J., Prausnitz, J.M. (1978). Vapor-liquid equilibria in multicomponent aqueous solutions of volatile weak electrolytes. AIChE. J. 24 (6), 966-976. https://doi.org/10.1002/aic.690240605.

García, P.A., Rojas-Vargas, A. (2006). Comparación de los floculantes CHEMADD A-41, 42 y 43 de la firma Chematek, el IP-600 de la firma Zar Cia. y el Percol, versión canadiense del MAGNAFLOC R-155. Tecnología Química $26(3), 41-47$.

Guo, X-Y., Huang, K., Zhang, D.-M. (2004). Preparation of basic carbonate particles in solution system of Ni(III)$\mathrm{NH}_{3}-\mathrm{CO}_{3}{ }^{2-}-\mathrm{H}_{2} \mathrm{O}$. T. Nonferr. Metal. Soc. China 14 (5), $1006-1011$.

Grosse, A.C., Dicinoski, G.W., Shaw, M.J., Haddad, P.R. (2003). Leaching and recovery of gold using ammoniacal thiosulfate leach liquors (A Review). Hydrometallurgy 69 (1-3), 1-21. https://doi.org/10.1016/S0304-386X(02)00169-X.

Hikita, H., Konishi, Y. (1981). Absorption of Sulfur Dioxide into Aqueous Sodium Carbonate Solutions and Desorption of Carbon Dioxide. Bulletin of University of Osaka Prefecture. Series A, Engineering and Natural Sciences 30 (1), 29-40. http://hdl.handle.net/10466/8344.
Hummel, W., Thoenen, T., Berner, U., Curti, E. (2014). The PSI/ Nagra Chemical Thermodynamic Database 12/07. PSI Report Nr. TM-44-14-05, Paul Scherrer Institut, Villigen, Switzerland, p. 35. https://www.psi.ch/sites/default/files/ import/les/DatabaseEN/PSI-Bericht\%252014-04_final_ druckerei.pdf

Irving, H., Williams, R.J.P. (1953). The stability of transitionmetal complexes. J. Chem. Soc. 0, 3192-3210. https://doi. org/10.1039/jr9530003192.

Jones, D., Moore, R. (2001). The application of the CESL nickel process to laterites. Cominco Engineering Services Ltd., Canada. https://www.teck.com/media/CESL-PublicationNickel-Process-Laterites-Alta-2001.pdf

Kazuyoshi, S., Masayoshi, H., Tatsuhico, E. (1974). Kinetics and Mechanism of the Ammonia Pressure Leaching of Lateritic Ore Containing Nickel. T. Jpn. I. Met. 15 (2), 121-128. https://doi.org/10.2320/matertrans1960.15.121.

Kotelnikova, N.E., Mikhailidi, A.M. (2012). Hydrate cellulose films and preparation of samples modified with nickel nanoand microparticles. Cell. Chem. Technol. 46 (1-2), 27-33. http://www.cellulosechemtechnol.ro/pdf/CCT1-2(2012)/ p.27-33.pdf.

Magaña Haynes, M.E., Rojas-Vargas, A. (2013). Rango de pH para destilar el licor de lixiviación carbonato amoniacal. Tecnología Química 33 (3), 200-205.

Magaña Haynes, M.E. (2016). Evaluación de la adición de licor con alto contenido de Amoniaco y Dióxido de Carbono en el alambique 208 de la Empresa Comandante Che Guevara. Tecnología Química 36 (1), 27-36.

Rojas-Vargas, A.. Trujillo, M.E. (2012). Medición del pH durante la destilación del licor producto del proceso CARON. Parte (III). Tecnología Química 32 (2), 177-185.

Schröder, D., Ducháčková, L., Tarábek, J., Karwowska, M., Fijalkowski, K., Ončák, M., Slavíček, P. (2011). Direct Observation of Triple Ions in Aqueous Solutions of Nickel(II) Sulfate: A Molecular Link Between the Gas Phase and Bulk Behavior. J. Am. Chem. Soc. 133 (8), 2444-2451. https://doi. org/10.1021/ja105408a.

Scott, W.D., Hobbs, P.V.(1967). The Formation of sulfate in water Droplets. Journal of the atmospheric Sciences 24, 54-57. https://doi.org/10.1175/1520-0469(1967)024<0054:TFOSI $\mathrm{W}>2.0 . \mathrm{CO} ; 2$.

Smith, R.M., Martell, A.E. (1976). Critical Stability Constants. Inorganic Complexes. Volume 4, Plenum Press, New York. https://doi.org/10.1007/978-1-4757-5506-0.

Söhnel, O., Mullin, J. (1979). Kinetics of precipitation of nickel ammonium sulphate hexahydrate from aqueous solutions. Cryst. Res. Technol. 14 (2), 217-228. https://doi.org/10.1002/ crat. 19790140215

Warner, T.E. (1988). An electrochemical study of the oxidative dissolution of synthetic nickel-iron-sulphide minerals in aqueous media. PhD Thesis, University of Leeds, Department of Mining and Mineral Engineer, p. 499. https://etheses.whiterose.ac.uk/1075/.

Xu, B., Kong, W., Li, Q., Yang, Y., Jiang, T., Liu, X. (2017). A Review of Thiosulfate Leaching of Gold: Focus on Thiosulfate Consumption and Gold Recovery from Pregnant Solution. Metals 7 (6), 1-16. https://doi.org/10.3390/met7060222.

Yoosefian, M., Ahmadzadeh, S., Aghasi, M., Dolatabadi, M. (2017). Optimization of electrocoagulation process for efficient removal of ciprofloxacin antibiotic using iron electrode; kinetic and isotherm studies of adsorption. J. Mol. Liq. 225, 544-553. https://doi.org/10.1016/j.molliq.2016.11.093.

Zhan, J., He, Y-H., Zhou, Di-F, Zhang, C.-F (2011). Thermodynamic analysis on synthesis of fibrous Ni-Co alloys precursor and Ni/Co ratio control. T. Nonferr. Metal. Soc. China 21 (5), 1141-1148. https://doi.org/10.1016/S10036326(11)60834-0.

Zhang, W., Cheng, C.Y. (2007). Manganese metallurgy review. Part I: Leaching of ores/secondary materials and recovery of electrolytic/chemical manganese dioxide. Hydrometallurgy 89 (3-4), 137-159. https://doi.org/10.1016/j. hydromet.2007.08.010. 